\title{
Properties of massive Population III and metal-poor stars
}

\author{
Daniel Schaerer ${ }^{1,2}$ \\ ${ }^{1}$ Geneva Observatory, 51 Ch. des Maillettes, CH-1290 Sauverny, Switzerland \\ email: daniel.schaerer@obs.unige.ch \\ ${ }^{2}$ Laboratoire d'Astrophysique, OMP, 14 Avenue E. Belin, F-31400 Toulouse, France
}

\begin{abstract}
We review the properties of massive Population III and very metal-poor stars, including briefly their formation, IMF, their main sequence evolution, possible mass loss mechanisms, atmosphere modeling etc. For detailed predictions concerning the properties of these stars we refer to Schaerer (2002) and Schaerer (2002) and references therein. Extending these calculations, Schaerer \& Fall (2007) present new calculations concerning the ionizing power, Ly- $\alpha$ strength and related properties for different metallicities as well as for a range of power-law and log-normal IMFs. For illustrations from these studies see the Figures below. New detailed calibrations for solar metallicity O-type stars have recently been presented by Martins et al. (2005).
\end{abstract}

Keywords. galaxies:high-redshift, stars: Population III, stars: general
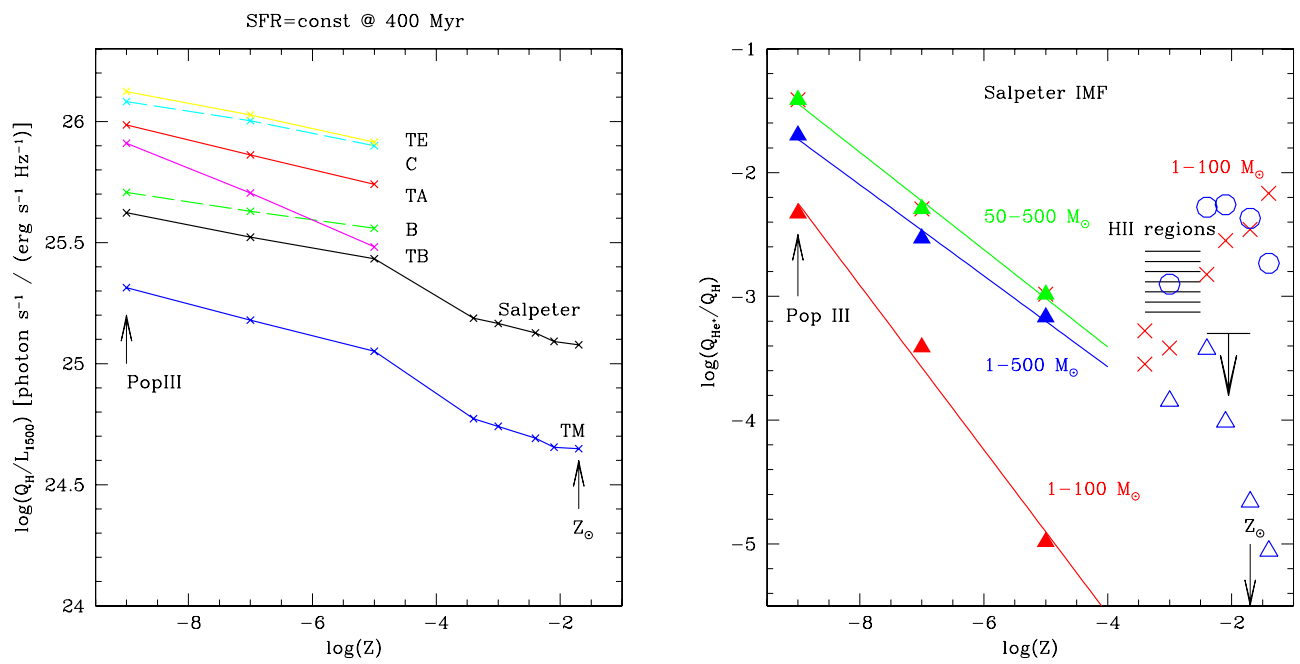

Figure 1. Left: Predicted ionizing to UV power of starbursts as a function of metallicity, from $Z \simeq 0$ (Pop.III) to solar. Different curves illustrate the predictions for various IMFs, including the Salpeter IMF and several log-normal IMFs (for details see Schaerer \& Fall 2007) Right: Predicted hardness (as measured by the ratio of the $\mathrm{He}^{+}$to $\mathrm{H}$ ionizing photons) of the ionizing spectrum of starbursts from Pop.III to normal metallicities (from Schaerer 2003).

\section{References}

Martins, F., Schaerer, D., \& Hillier, D. J. 2005, A\&A, 436, 1049

Schaerer, D. 2002, A\&A, 382, 28

Schaerer, D. 2003, $A \mathscr{E} A, 397,527$

Schaerer, D., \& Fall, M. 2007, in preparation 